allen diesen Versuchen lag nicht die geringste Veranlassung vor, gerade die Traubesche Apparatur zu benutzen; der Tropfmethode an sich anhaftende Mängel ließen das durchaus nicht wünschenswert erscheinen.

Selbst zu A scoli und Izar setzt sich J. Traube in bemerkenswerten Gegensatz; denu diese erkennen den von mir geschilderten Ent. wicklungsgang dieser Forschung rückhaltlos an. (Z. B. Izar Zeitschrift f. Immunitätsf. Orig. Bd. VII, S. 199.) (5)

Doch genug der unfruchtbaren Strejtigkeiten, die lediglich J. Tra u be heraufbeschworen hat. Wünscht er in dieser Forsching genannt zu werden, so nıuß er die oben beschriebene Fragestellung, und zwar experimentell bearbeiten, was wir natïrlich mit Freude begriißen würden. Sehr erwünscht wäre es noch, wenn er vor Abfassung eines hierher geliörigen Artikels, und sei dieser auch noch so kurz, die Entwicklung der einsehlägigen Inımunitätsliteratur etwas verfolgen wollte.

Sollte es aber J. Tra ube vorziehen, weiter mit ganz unbegründeten, lediglich herabsetzenden Ausdrücken wie ,undiskuttabel" zu operieren, so würde sich allerdings eine weitere Diskussion nit ilım für uns erledigen.

Liter at ur: 1. Chemikerzeitung 1908, No, 20. - 2. Berliner klinische Wochenschrift 1908, No. 20. - 3. Zweite Tagung der Freien Vereinigung far Mikrobiologie. Berlin 1908. Zentralblatt für Bakteriologie. Ref. S. 143. - 4. Physiologische Gesellschaft zu Berlin, 19. Februar 1909. Medizinische Klinik 1909, No. 12. - 5. Die übrige

\title{
Zu den Bemerkungen J. Traubes: „Zur Diagnose der Syphilis" in No. 5 dieser Wochenschrift.
}

\section{Von Dr. Friedr. Schroen.}

J. Traube verkennt, wie seine Prioritätsansprüche beweisen, das Wesen der in der letzten Zeit an biologischen Laboratorien mit Aussicht auf Erfolg bearbeiteten Reaktionen vollkommen.

Uns liegt es fern, die Verdienste J. Traubes schmälern zu wollen. Er fand zunächst in seinem Stalagmometer eine besondere Methode, Oberflächenspannungen zu messen. Diese Methode ist sehr bequem, ihre Genauigkeit wird von vielen Fachgenossen J. Traubes bestritten, auch werden wohl die meisten seiner weitgehenden Theoreme gerade von den Maßgebendsten auf physikalisch-chemischem Gebiete noch abgelehnt.

Traube machte, wie schon andere vor ihm, darauf aufmerksam, daß physikalisch-chemische Reaktionen auch biologisch von Bedeutung sein können. Er beruft sich auf seine Arbeiten in Pflügers Archiv, Bd. 15. Hier fülırt Traube in einer Anmerkung die gesprächsweise von ihm gehörte (!), jedem Biologen geläufige Tatsache an, daß Tetanustoxin in den Nervenbahnen, Tetanusantitoxin in der Blutbahn wandert, und knüpft daran die selbstverständliche Erörterung, daß sich diese beiden Substanzen auch physikalisch verschieden verhalten miissen. Irgendwelche Versuche werden nicht angegeben.

Ueber die Beziehungen der Toxine zu den Antitoxinen finden wir in den früheren Traubeschen Arbeiten nicht die geringste Andeutung. Das ist nun aber gerade das Wesen unserer Reaktionen. Dadurch werden sie diagnostisch wertvoll; denn Toxine an sich sind chemisch undefinierbare Gemische, das Antitoxin ist nur ein ganz kleiner Bruchteil des Serums. Ihre spezifische Beeinflussung, die sich in staunenerregend feiner Weise zeigt, wenn man ein geeignetes physikalisches System als Indikator einführt, macht das Wesen unserer Reaktion aus.

Diese spezifische Beeinflussung der verschiedensten Antigene durch ihre spezifischen Antikörper mit Hilfe bekann ter physikalischer Systeme zu demonstrieren, lag J. Traube ganz fern, und ich überlasse seine Prioritätsansprüche ruhig dem Urteile der Fachgenossen.

Als W. Weichardt im Jahre 1907 zum ersten Male wahrnahm, da $B$ Antigene und Antikörper in geeigneten Verdiinnungen Diffusionsbeschleunigung zueinander zeigen, verfolgte er dieses Phänomen mit den verschiedensten physikalischen Apparaten, die für die Demonstration geeignet waren, und suchte zunächst eine besonders empfindliche Anordnung ausfindig zu machen. Er verwandte Kapillaren (1), die chemische Wage (2), ein von ihm zur Demonstration konstruiertes Instrument, das Diffusiometer (3). Später führte er, da alle diese Apparate für quantitative Messungen zu schwerfällig waren, ein in der Oberflächenbildung begriffenes System (Baryum-Strontium-Schwefelsäure) ein und fand, daß dabei der Umschlagspunkt von Indikatoren bei Antigen-Antikörperbeeinflussung in bestimmten Verdünnungen verschoben wird (4). Bei 